\title{
Nonfullerene Small-Molecule Acceptors with Extended Optical Absorption Based on the "Spliced" Strategy for Organic Solar Cells
}

\author{
Di Zhou ${ }^{\mathrm{a}, \mathrm{b}}$ \\ Zhilin Liu ${ }^{\mathrm{a}, \mathrm{c}}$ \\ Dangqiang Zhu*a (D) \\ Xiyue Yuana,b \\ Xichang Bao ${ }^{a}$ \\ Renqiang Yang*a (D) \\ ${ }^{a}$ Qingdao Institute of Bioenergy and Bioprocess Technology, Chinese Academy of \\ Sciences, Qingdao, China \\ zhudq@qibebt.ac.cn; yangrq@qibebt.ac.cn \\ ${ }^{b}$ Center of Materials Science and Optoelectronics Engineering, University of Chinese \\ Academy of Sciences, Beijing, China \\ 'College of Materials Science and Engineering, Qingdao University of Science and \\ Technology, Qingdao, China
}

Received: 29.09.2019

Accepted after revision: 12.11.2019

DOI: 10.1055/s-0039-3402049; Art ID: om-19-0012-oa

License terms: (c)

(c) 2019. The Author(s). This is an open access article published by Thieme under the terms of the Creative Commons Attribution-NonDerivative-NonCommercial-License, permitting copying and reproduction so long as the original work is given appropriate credit. Contents may not be used for commercial purposes, or adapted, remixed, transformed or built upon. (https://creativecommons.org/licenses/by-nc-nd/4.0/).

Abstract How to broaden the optical absorption of photovoltaic materials is one of the key issues in the design of high-performance organic solar cells. Nowadays, the sunlight of $400-550 \mathrm{~nm}$ wavelength range is not effectively utilized for most small-molecule nonfullerene acceptors. In this work, we proposed the "spliced" strategy of combining the acceptor-donor-acceptor type narrow band-gap small molecules and wide-band-gap perylene diimide (PDI) moieties via a flexible alkyl chain linkage, which could give the superposition effect of the absorption spectra, and three small-molecule acceptors (S1, S2, and S3) were designed based on various end-capping groups with different electron withdrawing abilities. Encouragingly, the as-constructed molecules can well make use of $400-550 \mathrm{~nm}$ sunlight with two independent absorption regions. Meanwhile, the aggregation of S1 with a highly planar end-capping group was dominated by both the PDI unit and main skeleton, while S2 and S3 exhibited PDI-controlled aggregation. When fabricated into organic solar cells, S1-based devices achieved a superior efficiency of $3.41 \%$ in comparison with those of the other two. The poor photovoltaic performance could be attributed to severe PDI aggregation, which can hinder the charge transfer through the main skeletons. This work could provide a new perspective to modulate optical absorption through the spliced strategy.

Key words Organic solar cells, nonfullerene acceptor, spliced strategy, optical absorption, aggregation

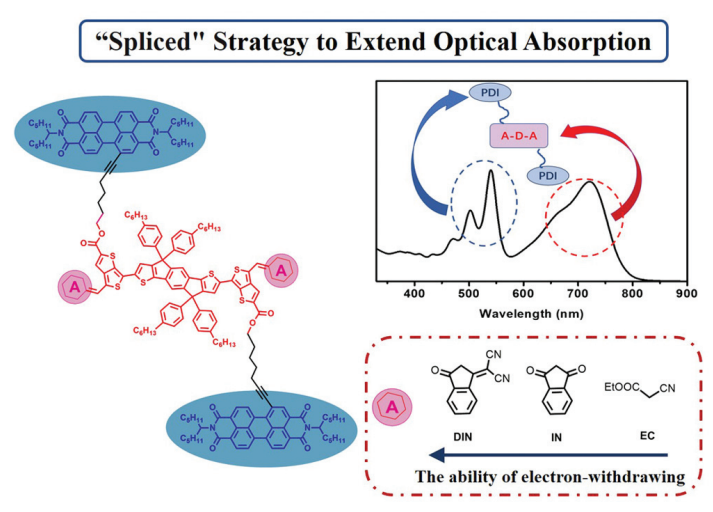

\section{Introduction}

Over the last few years, nonfullerene acceptors have attracted great interest in the field of organic solar cells (OSCs) due to their unique advantages of low cost, strong adsorption ability, and high thermal stability, and their photoelectric conversion efficiency (PCE) has increased significantly, inspiring researchers to conduct further research in terms of structure-property relationship and device architecture. ${ }^{1-10}$

One of the keys to the design of high-efficiency OSCs is to construct photovoltaic materials with wide and strong absorption in the visible-near-infrared region, which is in favor of the short-circuit current densities $\left(J_{s c}\right)$ in photovoltaic devices. ${ }^{11,12}$ Acceptor-donor-acceptor (A-D-A) type small-molecule acceptors (SMAs) are regarded as a class of promising semiconducting materials, which consist of two $\pi$-electron-withdrawing termini linked by a planar $\pi$-bridge consisting of fused rings. ${ }^{13-17}$ Since the first star acceptor ITIC was synthesized, a number of similar small molecules have been developed to regulate the energy levels and packing, such as IDIC, IEIC, ATT-1, etc. ${ }^{6,8,18,19}$ Hereinto, to match the solar spectrum as much as possible, many researchers have developed a series of design strategies to construct narrow band-gap (NBG) materials, such as extending the conjugated length and introducing strong electron-deficient end-capping groups or strong electronrich cores. ${ }^{20-23}$ What's more, the main absorption peaks of these NBG nonfullerene acceptors and some classical donor materials, such as PBDB-T and PTB7-Th, are generally located at $600-800 \mathrm{~nm}^{24-26}$ However, according to the spectrogram of sunlight, the energy at $400-550 \mathrm{~nm}$ accounts for nearly $30 \%$ of the total energy, as a result, the current materials could not completely utilize this part, 

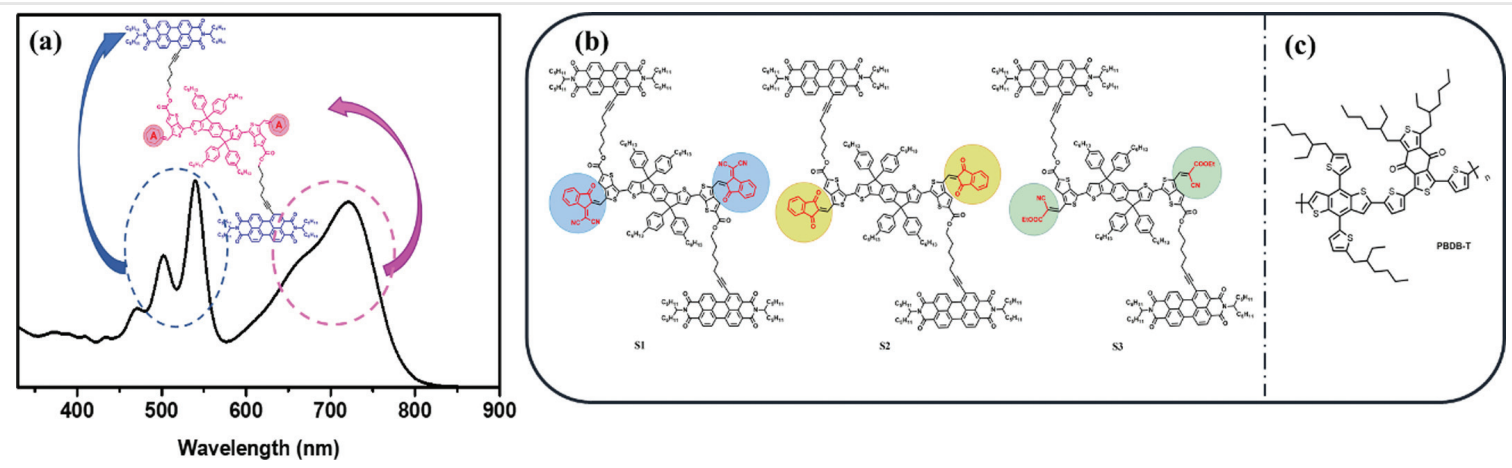

Figure 1 (a) Molecular structure and UV-vis absorption diagram. (b) Chemical structures of target acceptors. (c) The donor polymer PBDB-T.

which limits the probability of improvement in the photocurrent. ${ }^{27}$ On this basis, perylene diimide derivatives (PDIs) have attracted our attention due to their features of strong absorption at about $400-550 \mathrm{~nm}$, high electron mobility, and high environmental and thermal stability. In addition, the LUMO levels are generally located at approximately $-3.9 \mathrm{eV}$, similar to that of many A-D-A type acceptors. $^{28-32}$

Taking the above considerations into account, we proposed the "spliced" strategy of combining the traditional A-D-A type low-band-gap small molecules and wide-bandgap PDI moieties via a flexible alkyl-chain linkage, which could give the superposition effect of the absorption spectra. In this work, the A-D-A type electron acceptor, ATT-1 developed by Zhu, was chosen as the main backbone, which exhibits a broad absorption (500-800 nm), a large absorption coefficient, and a suitable LUMO energy level $(-3.63 \mathrm{eV}) .{ }^{8}$ In detail, three novel double-unit small molecules with indacenodithiophene (IDT) as the core, ${ }^{33-35}$ three different electron-accepting segments, 2-methylene-(3-(1,1-dicyanomethylene)-indanone) (IC), 1,3-indanedione (IN), and ethyl cyanoacetate (EC), as end-capping units, thieno[3,4-b]thiophene (TT) as the $\pi$-bridge and linking different terminal units and connecting PDI to TT through flexible unconjugated alkyl chains, named S1, S2, and S3, respectively, are shown in Figure 1, and the effects of electron-withdrawing endcapping groups on molecular stacking and photovoltaic performance were systematically studied. ${ }^{36}$

\section{Results and Discussion}

\section{Materials Synthesis and Characterization}

The synthesis routes of S1-S3 are demonstrated in Scheme S1. The TT derivative 3 was obtained via two steps of esterification reaction and classical Vilsmeier-Haack reaction, which could be further coupled with compound $\mathbf{4}$ to give compound 5. Subsequently, the PDI-based monomer $\mathbf{7}$ was obtained via the Sonogashira coupling reaction, which could give the target molecules S1S2S3 through Knoevenagel condensation with different end-capping groups. The target molecules could be readily dissolved in common solvents at room temperature, such as chloroform (CF), chlorobenzene (CB), dichloromethane (DCM). What's more, these molecules exhibited excellent thermal stability with decomposition temperature of $>300^{\circ} \mathrm{C}$ by thermal gravimetric analysis (TGA; Figure S1).

\section{Optical and Electrochemical Properties}

The linear ultraviolet absorption curves of S1, S2, and S3 are shown in Figures 2 and S2. The molecules showed a combination of absorption spectra of two types of acceptors. The high-energy region of about $450-600 \mathrm{~nm}$ (region I) could be attributed to that of PDI, ${ }^{37,38}$ while the low-energy region beyond $600 \mathrm{~nm}$ (region II) originated from the main skeleton. ${ }^{9}$ (a)

(c)
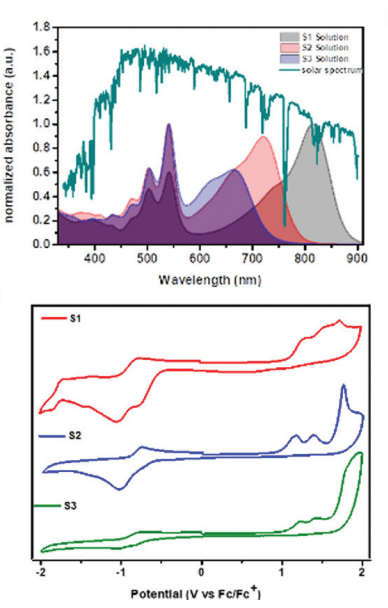

(b)

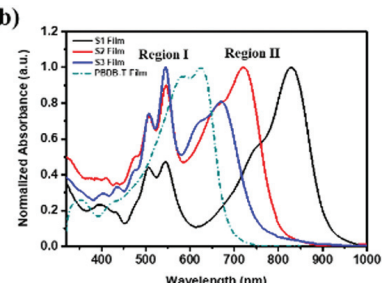

(d)

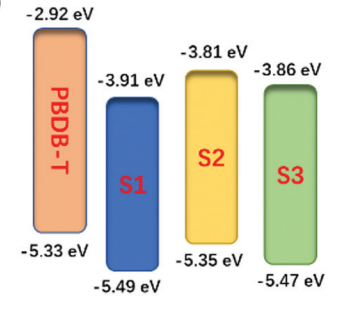

Figure 2 (a) Absorption spectra of S1, S2, and S3 in chloroform. (b) Film absorption spectra of SMAs and donors PBDB-T. (c) CV curves of S1, S2, and S3. (d) The energy band diagram. 
Table 1 Optical properties and frontier energy levels of three acceptors

\begin{tabular}{|c|c|c|c|c|c|c|}
\hline & $\begin{array}{l}\text { Solution } \\
\lambda_{\max }(\mathrm{nm})\end{array}$ & $\begin{array}{l}\text { Film } \\
\lambda_{\max }(\mathrm{nm})\end{array}$ & $\begin{array}{l}\text { Film } \\
\lambda_{\text {onset }}(\mathrm{nm})\end{array}$ & $\begin{array}{l}E_{\mathrm{g}}{ }^{\mathrm{opt}} \\
(\mathrm{eV})^{\mathrm{a}}\end{array}$ & $\begin{array}{l}\text { HOMO } \\
(\mathrm{eV})\end{array}$ & $\begin{array}{l}\text { LUMO } \\
(\mathrm{eV})\end{array}$ \\
\hline S1 & 815 & 830 & 920 & 1.35 & -5.49 & -3.91 \\
\hline S2 & 721 & 736 & 810 & 1.53 & -5.35 & -3.81 \\
\hline S3 & 540 & 545 & 764 & 1.62 & -5.47 & -3.86 \\
\hline
\end{tabular}

Obviously, the three acceptors have different ranges of absorption, caused by different end-capping units. With the increase of the withdrawing ability, the absorption gradually red-shifted. As shown in Figure 2a,b, S1 showed the widest absorption, with an absorption edge of $\sim 885 \mathrm{~nm}$ in solution, while the absorption edges of S2 and S3 were located at $\sim 794$ and $\sim 740 \mathrm{~nm}$, respectively, which implies the different electron withdrawing abilities of various endcapping units. By contrast, due to the enhanced molecular interactions, the absorption for the films had the red-shifts of 35,16 , and $24 \mathrm{~nm}$, respectively. Accordingly, the optical band gaps of S1-S3 were calculated to be $1.35,1.53$, and $1.62 \mathrm{eV}$, respectively. Molecular energy levels of the three SMAs determined by electrochemical cyclic voltammetry (CV) are shown in Figure $2 \mathrm{c}$ and Table 1, showing multiple oxidation and reduction peaks, which could be attributed to the two parts linked by flexible alkyl chains. ${ }^{39}$ According to the equation $E_{\mathrm{HOMO} / \mathrm{LUMO}}=-e\left(E_{\mathrm{ox} / \mathrm{red}}+4.4\right),{ }^{40}$ the HOMO energy levels of $\mathrm{S} 1, \mathrm{~S} 2$, and $\mathrm{S} 3$ were calculated to be -5.49 , -5.35 , and $-5.47 \mathrm{eV}$, respectively, which were similar to that of ATT-2. ${ }^{8}$ The LUMO energy levels of them were -3.91 , -3.81 , and $-3.86 \mathrm{eV}$, respectively, which were similar to those of PDIs. ${ }^{41}$ Compared with that of S1, S2 and S3 displayed relatively high LUMO energy levels, which were conducive to improve the $V_{\mathrm{OC}}$ of the devices.

In addition, the density functional theory (DFT) method under the B3LYP/6-31G(d,p) level was also utilized to analyze the energy level difference (Figure S3). The HOMO electron density is mainly delocalized on IDT and TT units of the main skeleton. By contrast, the LUMO energy level is mainly determined by PDI units for S2 and S3, but distributed on the whole A-D-A skeleton for S1, which also verifies the strongest electron withdrawing ability of the IC unit.

\section{Aggregation Behavior}

In order to study the aggregation behavior of small molecules, UV absorption in diluted solution and grazingincidence wide-angle X-ray scattering (GIWAXS) analysis was carried out. For the region I of the UV absorption spectrum (Figure 2b), the absorption intensities of S2 and S3 were much greater than that of S1, indicating that the aggregation of PDIs in S2 and S3 was stronger. For region II, Figures S2d and 3d show that the main skeleton absorption of the three small molecules becomes weaker in the absorption of the mixed solution, especially S2 and S3, which further verified that the molecular aggregation of S2 and S3 molecules was mainly through the PDI part. However, S1 was dominated by both PDI aggregation and skeleton aggregation. In addition, as the temperature increased, the small-molecule solution still showed a strong aggregation state, and the UV-vis absorption did not change substantially (Figure S2). In order to further explore the state of aggregation between molecules, the morphology of the three small molecules was studied using GIWAXS. As shown in Figure S4, the three molecules exhibited similar reflection at $\sim 15.5 \AA^{-1}$, corresponding to a $\pi-\pi$ stacking distance of $4.05 \AA$, obviously larger than

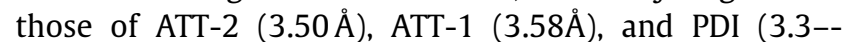
$3.5 \AA)^{8,9,28,41}$ The weakened stacking indicated the asconstructed molecules could not produce a synergistic effect on molecular aggregation. In addition, the neat films of S2 and S3 showed different (100) diffraction signals in the in-plane and out-of-plane directions, which indicated the disordered packing.

\section{Photovoltaic Performance}

In order to explore the photovoltaic properties of S1-S3, bulk heterojunction (BHJ) solar-cell devices were fabricated with the structure of ITO/PEDOT:PSS/donor:acceptor/ PDI$\mathrm{NO} / \mathrm{Al}$, where PEDOT:PSS is poly(3,4-ethylenedioxythiophene):poly(styrenesulfonate). In order to further complement each other on the absorption spectrum and expand the absorption range, PBDB-T with a strong absorption at 550- $670 \mathrm{~nm}$ was chosen as the donor material, which just filled the vacancy of the acceptors. ${ }^{25}$ The photovoltaic performance of solar cells under different conditions was investigated, as shown in Figure 3 and Table 2. The detailed data are shown in the Supporting Information (Table S1). Unfortunately, the devices based on the new small molecules exhibited poor performance, which could be caused by the low electron mobility originated from the PDI aggregation. However, the end-capping groups produce great influence on the photovoltaic performance. Compared 
(a)

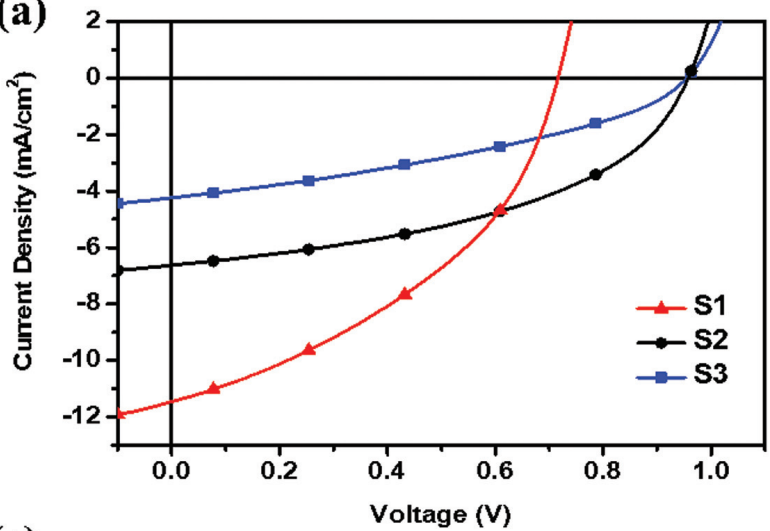

(c)

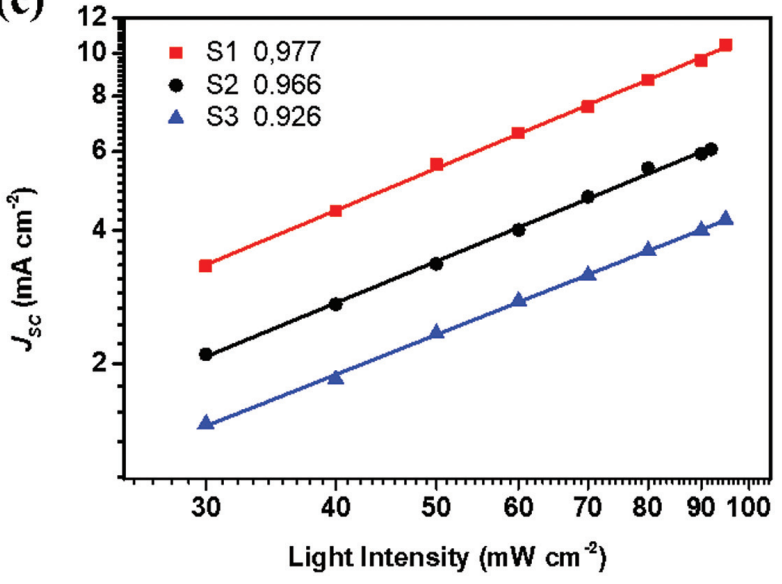

(b)

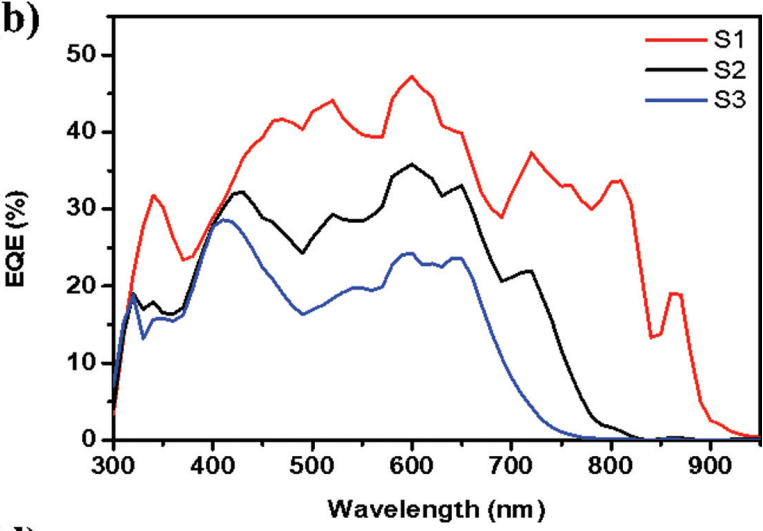

(d)

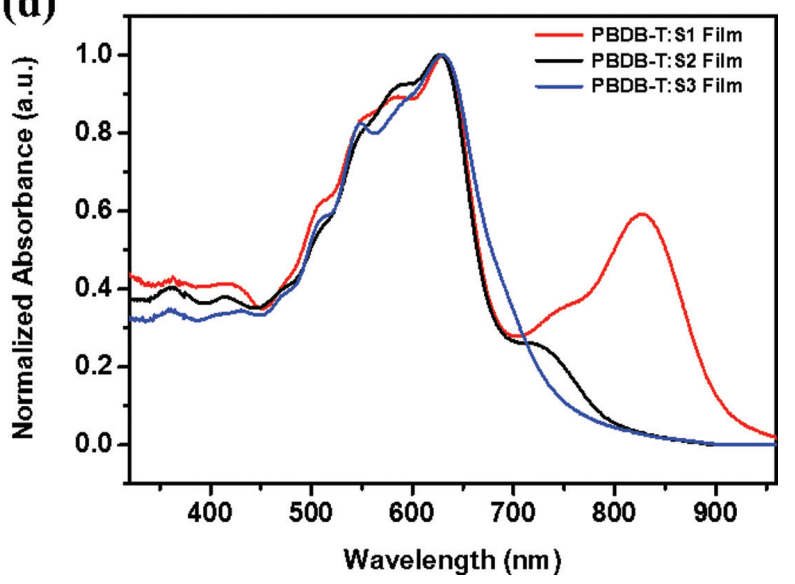

Figure 3 (a) The optimal J-V curves of PBDB-T:SMA-based devices. (b) EQE curves of the corresponding PSCs. (c) Jsc-light intensity plots of the OSCS under optimal conditions; the solid lines represent the corresponding fitted curves. (d) Absorption spectrum of mixed film.

with that of S1, S2- and S3-based devices exhibited obviously higher $V_{\mathrm{OC}}$ of $>0.90 \mathrm{~V}$ due to their relatively high LUMO energy levels. Meanwhile, the $J_{S C}$ values of S1based devices were superior to those of S2 and S3, which would be induced by the wider absorption and excellent blend film morphology. As a result, the S1-based devices exhibited the highest efficiency of $3.41 \%$ with a $V_{\mathrm{OC}}$ of $0.71 \mathrm{~V}$, a $J_{\mathrm{SC}}$ of $11.23 \mathrm{~mA} \mathrm{~cm}^{-2}$, and a FF of $42.23 \%$. The external quantum efficiencies (EQEs) of the optimal devices were investigated to prove the accuracy of the PCE measurement. As shown in Figure 3b, the polymer solar cells (PSCs) based on $\mathrm{S} 1$ showed the broadest photoresponse range from 300 to $900 \mathrm{~nm}$, which was in agreement with the corresponding absorption spectra of the blend films. As shown in Table 1, the integrated current densities $\left(J_{S C}{ }^{E Q E}\right)$ from the EQE spectra are roughly equivalent to the $J_{\mathrm{SC}}$ values obtained

Table 2 Device performance under different conditions

\begin{tabular}{|c|c|c|c|c|c|c|}
\hline & $\begin{array}{l}\text { Additive } \\
(\mathrm{v} / \mathrm{v})\end{array}$ & $\begin{array}{l}V_{\mathrm{OC}} \\
(\mathrm{V})\end{array}$ & $\begin{array}{l}\text { IsC }_{\mathrm{SC}} \\
\left.(\mathrm{mA} \mathrm{cm})^{-2}\right)\end{array}$ & $\begin{array}{l}\mathrm{FF} \\
(\%)\end{array}$ & $\begin{array}{l}\mathrm{PCE}_{\max } \\
\left(\mathrm{PCE}_{\text {ave }}\right)^{\mathrm{a}}(\%)\end{array}$ & $\begin{array}{l}\mu_{e} \\
\left(\mathrm{~cm}^{2} \mathrm{v}^{-1} \mathrm{~s}^{-1}\right)\end{array}$ \\
\hline PBDB-T:S1 & 1 & 0.73 & 7.71 & 39.03 & $2.21(2.16)$ & \\
\hline PBDB-T:S1 & $0.5 \% \mathrm{DPE}$ & 0.71 & 11.23 & 42.23 & 3.41 (3.39) & $1.11 \times 10^{-5}$ \\
\hline PBDB-T:S2 & 1 & 0.97 & 5.29 & 39.62 & $2.05(2.01)$ & \\
\hline PBDB-T:S2 & $0.5 \% \mathrm{CN}$ & 0.95 & 6.62 & 46.01 & $2.92(2.87)$ & $1.06 \times 10^{-5}$ \\
\hline PBDB-T:S3 & 1 & 0.91 & 2.93 & 31.54 & $0.84(0.82)$ & \\
\hline PBDB-T:S3 & $0.5 \% \mathrm{CN}$ & 0.95 & 4.23 & 36.64 & $1.47(1.45)$ & $1.17 \times 10^{-5}$ \\
\hline
\end{tabular}

average PCEs in brackets for 10 devices. 

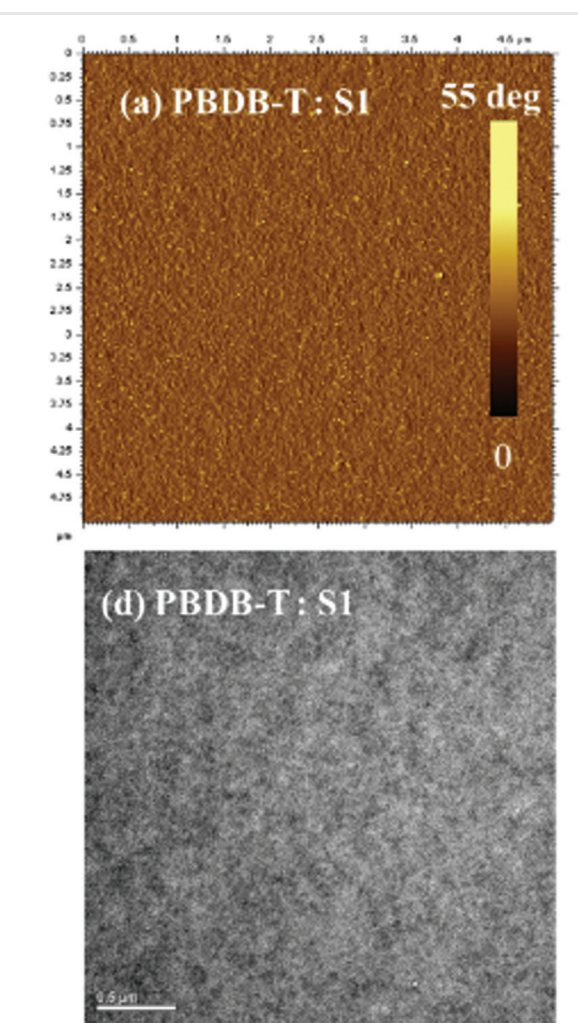
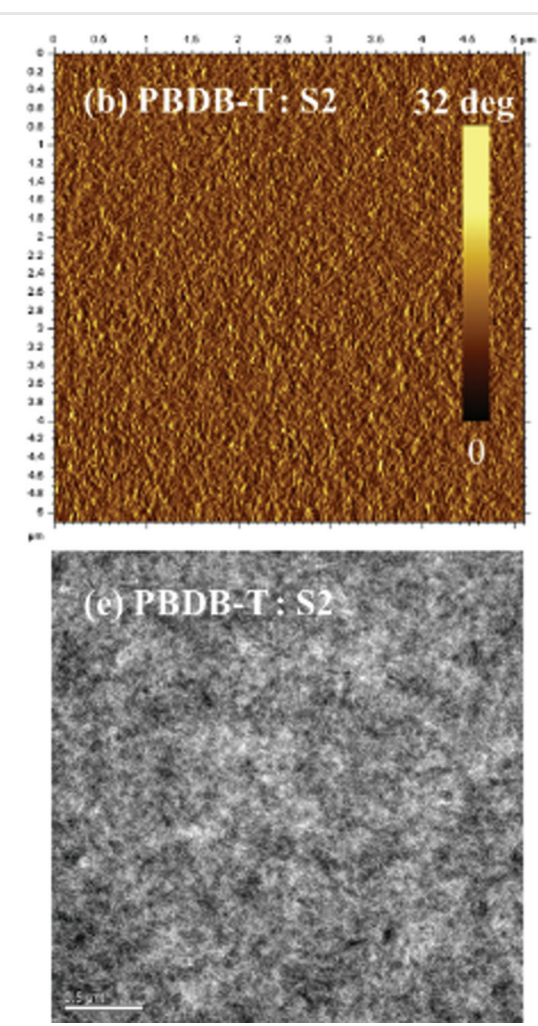
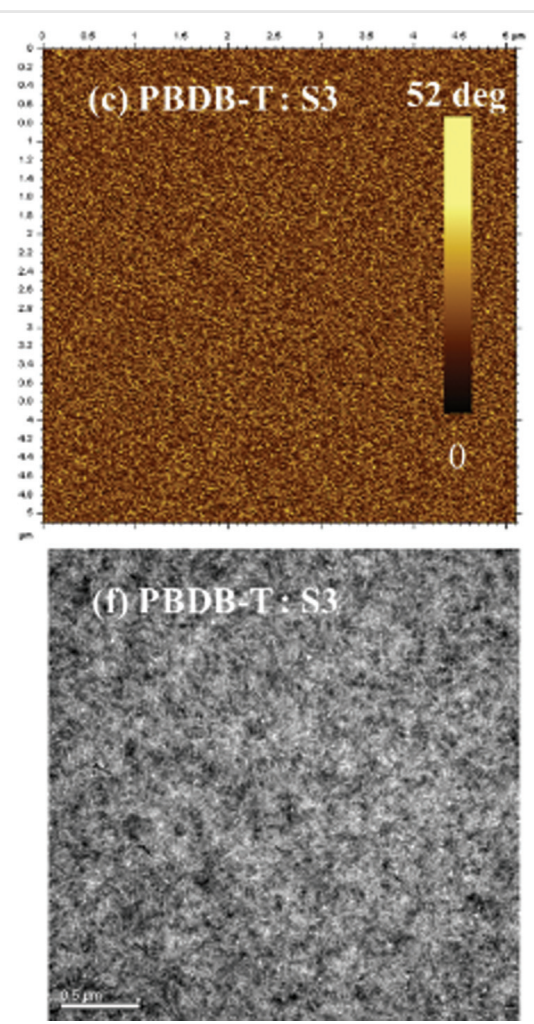

Figure 4 Tapping-mode AFM phase images of blend films: (a) PBDB-T:S1, (b) PBDB-T:S2, and (c) PBDB-T:S3. TEM images of (d) PBDB-T:S1, (e) PBDB-T: $\mathrm{S} 2$, and (f) PBDB-T:S3.

from the $J-V$ measurements. Furthermore, the electron mobilities of the optimal active layers were measured using the space-charge limited current (SCLC) model, as summarized in Table 1, and the plots of the current density versus voltage of devices based on SCLC are shown in Figure S5.

The three acceptor-based devices exhibited relatively low electron mobilities $\left(10^{-5} \mathrm{~cm}^{2} \mathrm{~V}^{-1} \mathrm{~s}^{-1}\right)$, which could be one of the main reasons for the low $J_{\mathrm{Sc}}$. Thus, it can be seen that the end-capping groups can control the optical absorption, the intermolecular aggregation mode, as well as the charge transfer. In order to further investigate the electron separation and recombination process in OSCs, the $J_{\mathrm{SC}}$ versus light intensity $(P)$ was plotted. Generally, $J_{S C}$ has a power-law dependence on the incident light power $\left(J_{\mathrm{Sc}} \propto P^{\alpha}\right.$.), The less the bimolecular recombination, the more the charge is collected by the electrode, the closer the $\alpha$ is to $1 .{ }^{42-44}$ As shown in Figure 3c, the $\alpha$ value of PBDB-T:S3 devices was 0.926, which indicated relatively strong bimolecular recombination under shortcircuit conditions and affected the $J_{S C}$ significantly. However, there are higher $\alpha$ values of 0.977 and 0.966 for PBDB-T:S1 and PBDB-T:S2 devices, respectively, suggesting that bimolecular recombination was suppressed for these devices.

\section{Molecular Packing and Morphology in Blends}

The microstructure of these blends was confirmed by atomic force microscopy (AFM) and transmission electron microscopy (TEM), as shown in Figures 4 and S6. From the AFM images of the blend films (Figures 4 and S6), we can find that all the blended films exhibited a smooth surface with small root-mean-square (RMS) values of $<2 \mathrm{~nm}$ (1.36 nm for PBDB-T:S1, $1.68 \mathrm{~nm}$ for PBDB-T:S2, and $1.60 \mathrm{~nm}$ for PBDB-T: S3). In addition, the acceptor S1 exhibited a uniform morphology, indicating good miscibility with PBDB-T. By contrast, with the decrease of main backbone aggregation, S2and S3-based active layers displayed an obvious wheat-like pattern because of the strong PDI aggregation. Furthermore, from TEM images, it was found that the morphology of PBDB-T:S1 exhibited a more ideal nanoscale that would be in favor of better charge separation and transport, resulting in the improvement of $J_{\mathrm{SC}}$.

\section{Conclusions}

In conclusion, three SMAs have been designed and synthesized via the "spliced" strategy to broaden the absorption range. They were formed by linking traditional 
A-D-A type narrow-band-gap small molecules and wideband-gap PDI moieties through alkyl chains. S2 and S3 gave higher LUMO levels, leading to a high $V_{\mathrm{OC}}$ of $0.95 \mathrm{~V}$ in the corresponding devices. The absorption edge of $\mathrm{S} 1$ reached $\sim 920 \mathrm{~nm}$, which made the corresponding device achieve the highest $J_{\mathrm{SC}}\left(11.23 \mathrm{~mA} \mathrm{~cm}^{-2}\right)$ and PCE (3.41\%). The aggregation modes of the three acceptors were different. S2 and S3 exhibited PDI-controlled aggregation while S1 with a highly planar end-capping group was dominated by both PDI aggregation and skeleton aggregation. Severe PDI aggregation can hinder the charge transfer between the main skeletons, resulting in low $J_{\mathrm{Sc}}$. Therefore, adjusting the PDI aggregation state will become the focus of the next step. In general, this work could provide a new perspective to modulate optical absorption through the spliced strategy.

\section{Experimental Section}

\section{Materials}

Compound 1 was synthesized through the reported method. ${ }^{45}$ Compounds $\mathbf{4 , 6}$, and the polymer donor PBDB-T were purchased from SunaTech Inc. Toluene (PhMe) was distilled over sodium in the presence of benzophenone as an indicator. $\mathrm{CHCl}_{3}$ and $\mathrm{DCM}$ were distilled over calcium hydride. The other reagents were purchased from commercial suppliers, which were used directly unless stated otherwise. The detailed synthetic processes of the small molecules are presented in the Supporting Information. Molecular structures were confirmed by ${ }^{1} \mathrm{H}-\mathrm{NMR},{ }^{13} \mathrm{C}-\mathrm{NMR}$, and MALDI-TOF (Figures S7-S13).

\section{Solar Cell Fabrication and Characterization}

Polymer solar-cell devices were fabricated with a conventional structure of ITO/PEDOT:PSS/polymer:SMA/ PDINO/Al. The patterned ITO glass was precleaned in an ultrasonic bath of acetone and isopropyl alcohol and treated in an ultravioletozone chamber (PREEN II-862) for $6 \mathrm{~min}$. Then a thin layer (about $30 \mathrm{~nm}$ ) of PEDOT:PSS was spin-coated onto the ITO glass at $4,000 \mathrm{rpm}$ and baked at $160^{\circ} \mathrm{C}$ for $15 \mathrm{~min}$. Solutions of polymer/SMA in CB $(10 \mathrm{mg} / \mathrm{mL})$ were stirred for 4 hours before spin-coating on the PEDOT:PSS layer to form the active layer of about $120 \mathrm{~nm}$ thickness. The thickness of the active layer was measured using a Veeco Dektak 150 profilometer. Then PDINO solution (in $\mathrm{CH}_{3} \mathrm{OH}, 1 \mathrm{mg} / \mathrm{mL}$ ) was spin-coated at 3,000 rpm to form the electron transport layer. Finally, an $\mathrm{Al}(\sim 100 \mathrm{~nm})$ metal electrode was thermally evaporated under about $4 \times 10^{-4} \mathrm{~Pa}$ and the device area was $0.1 \mathrm{~cm}^{2}$ defined by a shadow mask. The current density-voltage $(J-V)$ characteristics were measured with a Keithley 2400 source measure- ment unit under simulated $100 \mathrm{~mW} \mathrm{~cm}^{-2}$ irradiation from a Newport solar simulator. The EQEs were obtained using a certified Newport incident photon-to-current conversion efficiency (IPCE) measurement system.

\section{Instrumentation}

${ }^{1} \mathrm{H}$ and ${ }^{13} \mathrm{C}$ NMR spectra were recorded on a Bruker AVANCE-III 600 spectrometer using $\mathrm{CDCl}_{3}$ as the solution and tetramethylsilane (TMS) as the internal standard. TGA measurement was performed on STA-409 at a heating rate of $10{ }^{\circ} \mathrm{C} \mathrm{min}^{-1}$ under a nitrogen atmosphere. The UV-vis absorption spectrum was measured with a Hitachi U-4100 spectrophotometer. CV data were obtained using a CHI660D electrochemical workstation with a three-electrode cell consisting of a saturated calomel reference electrode, a carbon working electrode, and a platinum wire counter electrode. TEM images were obtained by using a HITACHI H-7650 electron microscope operating under an acceleration voltage of $100 \mathrm{kV}$. AFM images were obtained using an Agilent 5400 scanning probe microscope in the tapping mode. GIWAXS pattern measurements were performed at the beamline BL16B1 (Shanghai Synchrotron Radiation Facility). DFT was used under the B3LYP/6-31G (d,p) level.

\section{Funding Information}

The authors are deeply grateful to the National Natural Science Foundation of China (51573205 and 51773220) and the Youth Innovation Promotion Association CAS (2019215), for financial support.

\section{Acknowledgment}

The authors sincerely thank Dr. Chunming Yang from Shanghai Synchrotron Radiation Facility for GIWAXS measurement and Zezhou Liang from Lanzhou Jiaotong University for theoretical calculation.

\section{Supporting Information}

Supporting information for this article is available online at https://doi.org/10.1055/s-0039-3402049.

\section{References}

(1) Meng, L.; Zhang, Y.; Wan, X.; Li, C.; Zhang, X.; Wang, Y.; Ke, X.; Xiao, Z.; Ding, L.; Xia, R.; Yip, H.-L.; Cao, Y.; Chen, Y. Science 2018 , $361,1094$. 
(2) Yuan, J.; Zhang, Y.; Zhou, L.; Zhang, G.; Yip, H. L.; Lau, T. K.; Lu, X.; Zhu, C.; Peng, H.; Johnson, P. A.; Leclerc, M.; Cao, Y.; Ulanski, J.; Li, Y.; Zou, Y. Joule 2019, 3, 1140.

(3) Hou, J.; Inganäs, O.; Friend, R. H.; Gao, F. Nat. Mater. 2018, 17, 119.

(4) Liang, Z.; Tong, J.; Li, H.; Wang, Y.; Wang, N.; Li, J.; Yang, C.; Xia, Y. J. Mater. Chem. A 2019, 7, 15841.

(5) Yan, C.; Barlow, S.; Wang, Z.; Yan, H.; Jen, A. K. Y.; Marder, S. R.; Zhan, X. Nat. Rev. Mater. 2018, 3, 18003.

(6) Lin, Y.; Wang, J.; Zhang, Z. G. Adv. Mater. 2015, 27, 1170.

(7) Liang, Z.; Tong, J.; Li, H.; Wang, Y.; Wang, N.; Li, J.; Yang, C.; Xia, Y. J. Mater. Chem. A 2019, 7, 15841.

(8) Liu, F.; Zhou, Z.; Zhang, C.; Vergote, T.; Fan, H.; Liu, F.; Zhu, X. J. Am. Chem. Soc. 2016, 138, 15523.

(9) Liu, F.; Zhou, Z.; Zhang, C.; Zhang, J.; Hu, Q.; Vergote, T.; Liu, F.; Russell, T. P.; Zhu, X. Adv. Mater. 2017, 29, 1606574.

(10) Wang, X.; Du, Z.; Dou, K.; Jiang, H.; Gao, C.; Han, L.; Yang, R. Adv. Energy Mater. 2019, 9, 1802530.

(11) Li, Y. Acc. Chem. Res. 2012, 45, 723.

(12) Huang, W.; Cheng, P.; Yang, Y. M.; Li, G.; Yang, Y. Adv. Mater. 2018, 30, 1705706.

(13) Li, T.; Dai, S.; Ke, Z.; Yang, L.; Wang, J.; Yan, C.; Ma, W.; Zhan, X. Adv. Mater. 2018, 30, 1705969.

(14) Wang, W.; Yan, C.; Lau, T. K.; Wang, J.; Liu, K.; Fan, Y.; Lu, X.; Zhan, X. Adv. Mater. 2017, 29, 1606574.

(15) Yao, Z.; Liao, X.; Gao, K.; Lin, F.; Xu, X.; Shi, X.; Zou, L.; Liu, F.; Chen, Y.; Jen, A. K. J. Am. Chem. Soc. 2018, 140, 2054.

(16) Chen, F. X.; Xu, J. Q.; Liu, Z. X.; Chen, M.; Xia, R.; Yang, Y.; Lau, T. K.; Zhang, Y.; Lu, X.; Yip, H. L.; Jen, A. K.; Chen, H.; Li, C. Z. Adv. Mater. 2018, 30, 1803769.

(17) Xiao, B.; Tang, A.; Zhang, J.; Mahmood, A.; Wei, Z.; Zhou, E. Adv. Energy Mater. 2017, 7, 1602269.

(18) Lin, Y.; He, Q.; Zhao, F.; Huo, L.; Mai, J.; Lu, X.; Su, C. J.; Li, T.; Wang, J.; Zhu, J.; Sun, Y.; Wang, C.; Zhan, X. J. Am. Chem. Soc. 2016, 138, 2973.

(19) Lin, Y.; Zhang, Z.-G.; Bai, H.; Wang, J.; Yao, Y.; Li, Y.; Zhu, D.; Zhan, X. Energy Environ. Sci. 2015, 8, 610.

(20) Xiao, Z.; Jia, X.; Li, D.; Wang, S.; Geng, X.; Liu, F.; Chen, J.; Yang, S.; Russell, T. P.; Ding, L. Sci. Bull. 2017, 62, 1494.

(21) Xue, P.; Xiao, Y.; Li, T.; Dai, S.; Jia, B.; Liu, K.; Wang, J.; Lu, X.; Han, R. P. S.; Zhan, X. J. Mater. Chem. A 2018, 6, 24210.

(22) Yao, H.; Cui, Y.; Yu, R.; Gao, B.; Zhang, H.; Hou, J. Angew. Chem. Int. Ed. 2017, 56, 3045.

(23) Zhao, W.; Li, S.; Yao, H.; Zhang, S.; Zhang, Y.; Yang, B.; Hou, J. J. Am. Chem. Soc. 2017, 139, 7148.

(24) Liao, S. H.; Jhuo, H. J.; Cheng, Y. S.; Chen, S. A. Adv. Mater. 2013, $25,4766$.
(25) Zhao, W.; Qian, D.; Zhang, S.; Li, S.; Inganas, O.; Gao, F.; Hou, J. Adv. Mater. 2016, 28, 4734.

(26) Qian, D.; Ye, L.; Zhang, M.; Liang, Y.; Li, L.; Huang, Y.; Guo, X.; Zhang, S.; Tan, Z. a.; Hou, J. Macromolecules 2012, 45, 9611.

(27) Liu, T.; Guo, Y.; Yi, Y.; Huo, L.; Xue, X.; Sun, X.; Fu, H.; Xiong, W.; Meng, D.; Wang, Z.; Liu, F.; Russell, T. P.; Sun, Y. Adv. Mater. 2016, 28, 10008.

(28) Sun, D.; Meng, D.; Cai, Y.; Fan, B.; Li, Y.; Jiang, W.; Huo, L.; Sun, Y.; Wang, Z. J. Am. Chem. Soc. 2015, 137, 11156.

(29) Zhang, J.; Li, Y.; Huang, J.; Hu, H.; Zhang, G.; Ma, T.; Chow, P. C. Y.; Ade, H.; Pan, D.; Yan, H. J. Am. Chem. Soc. 2017, 139, 16092.

(30) Liu, X.; Du, X.; Wang, J.; Duan, C.; Tang, X.; Heumueller, T.; Liu, G.; Li, Y.; Wang, Z.; Wang, J.; Liu, F.; Li, N.; Brabec, C. J.; Huang, F.; Cao, Y. Adv. Energy Mater. 2018, 8, 1801699.

(31) Liu, Z.; Wu, Y.; Zhang, Q.; Gao, X.J. Mater. Chem. A 2016, 4, 17604.

(32) Liang, N.; Meng, D.; Ma, Z.; Kan, B.; Meng, X.; Zheng, Z.; Jiang, W.; Li, Y.; Wan, X.; Hou, J.; Ma, W.; Chen, Y.; Wang, Z. Adv. Energy Mater. 2017, 7, 1601664.

(33) Tang, A.; Xiao, B.; Wang, Y.; Gao, F.; Tajima, K.; Bin, H.; Zhang, Z. G.; Li, Y.; Wei, Z.; Zhou, E. Adv. Funct. Mater. 2018, 28, 1704507.

(34) Tang, A.; Xiao, B.; Chen, F.; Zhang, J.; Wei, Z.; Zhou, E. Adv. Energy Mater. 2018, 8, 1801582.

(35) Tang, A.; Song, W.; Xiao, B.; Guo, J.; Min, J.; Ge, Z.; Zhang, J.; Wei, Z.; Zhou, E. Chem. Mater. 2019, 31, 3941.

(36) Ni, W.; Wan, X.; Li, M.; Wang, Y.; Chen, Y. Chem. Commun. 2015, $51,4936$.

(37) Luo, Z.; Liu, T.; Chen, Z.; Xiao, Y.; Zhang, G.; Huo, L.; Zhong, C.; Lu, X.; Yan, H.; Sun, Y.; Yang, C. Adv. Sci. 2019, 6, 1802065.

(38) Zhang, X.; Jiang, B.; Zhang, X.; Tang, A.; Huang, J.; Zhan, C.; Yao, J. J. Phys. Chem. C 2014, 118, 24212.

(39) Lai, W.; Li, C.; Zhang, J.; Yang, F.; Colberts, F. JM.; Guo, B.; Wang, Q. M.; Li, M.; Zhang, R. A.; Janssen, J.; Zhang, M.; Li, W. Chem. Mater. 2017, 29, 7073.

(40) Zhu, D.; Bao, X.; Ouyang, D.; Wang, J.; Yuan, X.; Wang, Q.; Zhou, D.; Wen, S.; Yang, R. Nano Energy 2017, 40, 495.

(41) Zhang, J.; Bai, F.; Li, Y.; Hu, H.; Liu, B.; Zou, X.; Yu, H.; Huang, J.; Pan, D.; Ade, H.; Yan, H. J. Mater. Chem. A 2019, 7, 8136.

(42) Li, J.; Liang, Z.; Wang, Y.; Li, H.; Tong, J.; Bao, X.; Xia, Y. J. Mater. Chem. C 2018, 6, 11015.

(43) Uddin, M. A.; Lee, T. H.; Xu, S.; Park, S. Y.; Kim, T.; Song, S. Nguyen, T. L.; Ko, S. J.; Hwang, S.; Kim, J. Y.; Woo, H. Y. Chem. Mater. 2015, 27, 5997.

(44) Wang, Y.; Liang, Z.; Li, X.; Qin, J.; Ren, M.; Yang, C.; Bao, X.; Xia, Y.; Li, J. J. Mater. Chem. C 2019, 7, 11152.

(45) Zhu, D.; Wang, Q.; Wang, Y.; Bao, X.; Qiu, M.; Shahid, B.; Li, Y.; Yang, R. Chem. Mater. 2018, 30, 4639. 